Pacific Journal of Mathematics

HOMOGENIZATION OF REGULAR RINGS OF BOUNDED 


\title{
HOMOGENIZATION OF REGULAR RINGS OF BOUNDED INDEX, II
}

\author{
JOHN HANNAH
}

\begin{abstract}
Recently Goodearl and Handelman (2) derived sufficient conditions for a regular ring of bounded index to be a direct product of homogeneous regular rings. In this note we show how their conditions may be modified to yield necessary and sufficient conditions. We also discuss the extension of these results to semisimple $\pi$-regular rings of bounded index.
\end{abstract}

We shall follow the terminology of [3] and [4]. In particular rings are always associative but need not have a multiplicative identity. We say that a ring $R$ has bounded index if there is a positive integer $n$ such that $a^{n}=0$ for each nilpotent element $a$ of $R$. The least such $n$ is called the index of $R$.

Let $R$ be a regular ring with index $n$. Kaplansky has shown in [4, Theorem 2.3] that if $P$ is any primitive ideal of $R$ then $R / P$ is a simple Artinian ring of length at most $n$. (Here the length of a simple Artinian ring $S$ is just the length of a composition series for the module $S_{S}$.) This led Kaplansky to consider how $R$ may be constructed from certain 'homogeneous' parts. He says that a regular ring is homogeneous if all its primitive factor rings are simple Artinian of the same length. A homogeneous ring with an identity element is just a full matrix ring over a strongly regular ring [4, Theorem 4.2]. Furthermore $R$ can be built up from homogeneous rings by means of a finite number of ring extensions: for each $k$ let $I_{k}$ denote the intersection of all primitive ideals $P$ of $R$ for which $R / P$ has length at most $k$; then $I_{n}=0$ and each $I_{k-1} / I_{k}$ is homogeneous (see $[4, \S 4]$ ). However $R$ need not be a direct product of these homogeneous rings (see [2, page 64] and [4, page 70] for examples). We shall be considering the complementary picture of $R$ which may be obtained by using another family of ideals of $R$. For each $k$ let $J_{k}$ be the intersection of those primitive ideals $P$ of $R$ for which $R / P$ has length at least $k$. The semisimplicity of $R$ now ensures that $J_{1}=0$ and [4, Theorem 2.3] ensures that $J_{n+1}=R$. This time however the rings $J_{k+1} / J_{k}$ need not be homogeneous (the examples in [2, page 64] and [4, page 70] show this). Indeed we shall prove (in Theorem 2) that $R$ is a direct product of homogeneous rings if and only if each $J_{k+1} / J_{k}$ is homogeneous. We then use some results of Goodearl and Handelman [2] to relax this condition on $J_{k+1} / J_{k}$. For example if $R$ is also biregular it turns out that the length of 
a primitive factor ring of $J_{k+1} / J_{k}$ always divides $k$ anyway. Thus in this case we just need to assume in Theorem 2 that the length of a primitive factor ring of $J_{k+1} / J_{k}$ is never a proper divisor of $k$ (Theorem 4). A similar, but more complicated, criterion can be used if $R$ has an identity (Theorem 3). The sufficiency results in [2] are easy consequences of these theorems.

We begin with a result (due to Kaplansky) which relates the primitive factor rings of $J_{k+1} / J_{k}$ to those of $R$.

Lemma 1. Suppose $R$ is a regular ring of bounded index. Let $I$ be an ideal of $R$ and $Q$ an ideal of $I$. Then $Q$ is a primitive ideal of $I$ if and only if there is a primitive ideal $P$ of $R$ such that $I \varsubsetneqq P$ and $Q=I \cap P$. Furthermore when this is the case the rings $I / Q$ and $R / P$ are isomorphic.

Proof. The first statement is proved in [3, page 206] and [4, Theorem 3.1]. For the second statement it is enough to notice that $I / Q$ and $R / P$ are simple Artinian rings (by [4, Theorem 2.3]) and that $I / Q$ is isomorphic to the ideal $I+P / P$ of $R / P$.

This lemma implies that $J_{k}$ is the same whether it was defined as an ideal of $R$ or of $J_{m}$ where $m>k$. More precisely, if we write Spec $S$ for the set of primitive ideals of a ring $S$, then for each $m>k$ we have

$$
\begin{aligned}
J_{k}= & \cap\{P \in \operatorname{Spec} R: R / P \text { has length } \geqq k\} \\
= & \cap\{P \in \operatorname{Spec} R: R / P \text { has length } \geqq m\} \\
& \cap \cap\{P \in \operatorname{Spec} R: R / P \text { has length } \geqq k\} \\
= & J_{m} \cap \cap\left\{P \in \operatorname{Spec} R: J_{m} \varsubsetneqq P \text { and } R / P \text { has length } \geqq k\right\} \\
= & \cap\left\{J_{m} \cap P: P \in \operatorname{Spec} R, J_{m} \nsubseteq P \text { and } R / P \text { has length } \geqq k\right\} \\
= & \cap\left\{Q \in \operatorname{Spec} J_{m}: J_{m} / Q \text { has length } \geqq k\right\}
\end{aligned}
$$

as claimed.

THEOREM 2. Let $R$ be a regular ring of index $n$ and for each $k$ write

$$
J_{k}=\cap\{P \in \operatorname{Spec} R: R / P \text { has length } \geqq k\} .
$$

Then $R$ is a direct product of homogeneous rings if and only if each of the rings $J_{k+1} / J_{l}$ is homogeneous.

Proof. The necessity of this condition is straightforward to check. We prove the sufficiency by induction on $n$. If $n=1$ then $R$ is already homogeneous [4, page 67] so suppose the result is true 
for rings of index at most $n-1$ where $n>1$.

By [4, Theorem 2.3] we have $J_{n+1}=R$ and so our hypotheses imply that $R / J_{n}$ is homogeneous. Since $R / J_{n}$ has a primitive factor ring of length $n$ (as may be seen by choosing $P \in \operatorname{Spec} R$ such that $a^{n-1} \notin P$, where $a \in R$ with $a^{n}=0$ and $a^{n-1} \neq 0$ ), it follows that every primitive factor ring of $R / J_{n}$ has length $n$. Let

$$
J=\cap\{P \in \operatorname{Spec} R: R / P \text { has length }<n\}
$$

so that $J \cap J_{n}=\cap \operatorname{Spec} R=0$ since $R$ is semisimple. Suppose that $J+J_{n} \neq R$. Since $R$ is regular so is $R /\left(J+J_{n}\right)$ and so there is some primitive ideal $P$ of $R$ such that $J+J_{n} \leqq P$. But then $R / P$ is a primitive factor ring of both $R / J_{n}$ and $R / J$. The former implies that $R / P$ has length $n$, but the latter (since $R / J$ has index at most $n-1$ ) implies that $R / P$ has length at most $n-1$ (by [4, Theorem 2.3]). This contradiction shows that $J+J_{n}=R$.

Now $J \cong R / J_{n}$ is homogeneous. Furthermore $J_{n} \cong R / J$ has index at most $n-1$ and the remark after Lemma 1 thus shows that we can apply the induction hypothesis to $J_{n}$. Hence $R$ is a direct product of homogeneous rings.

REMARKs. (1) Since Kaplansky's original paper actually dealt with semisimple $\pi$-regular rings, rather than regular rings, it is probably worthwhile to indicate what scope there is for generalizing Theorem 2 in this direction. In fact Theorem 2 remains true if $R$ is a semisimple $\pi$-regular ring none of whose factor rings is a radical ring (the crucial point in the above proof being the existence of a primitive ideal $P$ containing $J+J_{n}$; see [3, Proposition 2, page 208]). Thus, for instance, Theorem 2 is still true if $R$ is a semisimple $\pi$-regular ring having an identity element. The following example, however, shows that Theorem 2 is not true for arbitrary semisimple $\pi$-regular rings. Choose a field $F$ and let $S=M_{2}(F)$ and $T=M_{3}(F)$, the $2 \times 2$ and $3 \times 3$ matrix rings over $F$ (respectively). Let $R$ be the ring of sequences of elements of $S \times T$ which are eventually constant and of the form

$$
\left[\left[\begin{array}{ll}
0 & a \\
0 & 0
\end{array}\right], \quad\left[\begin{array}{lll}
0 & 0 & a \\
0 & 0 & 0 \\
0 & 0 & 0
\end{array}\right]\right] \text { where } a \in F .
$$

Then $R$ is a semisimple $\pi$-regular ring of index 3 and each $J_{k+1} / J_{k}$ is homogeneous (see [4, Remark 2, page 69] for a similar example). However, any ideal of $R$ containing an element of the above form with $a \neq 0$ has primitive factor rings of length 2 and of length 3 . 
Hence $R$ cannot be a direct product of homogeneous rings.

(2) Goodearl and Handelman prove many of their results for regular rings whose primitive factor rings are all Artinian rather than just those of bounded index. However Theorem 2 does not extend to this generality even if $R$ has an identity (consider, for instance, the subalgebra of $\prod_{n} M_{n}(F)$ generated by $\bigoplus_{n} M_{n}(F)$ and the identity, where $F$ is a field). It should be noted though that if a regular ring whose primitive factor rings are all Artinian is a direct product of homogeneous rings it is a finite such direct product and so has finite index anyway (see [1, Theorem 6.2 and the remark following it]).

When the ring in Theorem 2 has an identity we can relax the conditions on $J_{k+1} / J_{k}$ somewhat.

THEOREM 3. Let $R$ be a regular ring with identity and suppose that $R$ has bounded index. The following conditions are equivalent.

(a) $R$ is a direct product of homogeneous rings.

(b) For each $k$, if $J_{k+1} / J_{k}$ has primitive factor rings of lengths $k_{1}, k_{2}, \cdots, k_{t}$ such that $m_{1} k_{1}+\cdots+m_{t} k_{t}=k$ for suitable positive integer's $m_{1}, m_{2}, \cdots, m_{t}$ then $t=1$ and $k_{1}=k$.

Proof. $\quad(a) \Rightarrow(b)$ is clear. Since a direct application of Theorem 2 does not seem to be possible here, we once more use induction on $n$, the index of $R$, to show that $(\mathrm{b}) \Rightarrow(\mathrm{a})$. We have $J_{n+1}=R$ and

$\cap\left\{P \in \operatorname{Spec}\left(R / J_{n}\right):\left(R / J_{n}\right) / P\right.$ has length $\left.n\right\}=0$.

Hence [2, Lemma 4] and our assumption (b) imply that $R / J_{n}$ is homogeneous. The argument in Theorem 2 now shows that $R=$ $J \oplus J_{n}$ where $J$ is homogeneous and $J_{n}$ has index at most $n-1$. Since $J_{n}$ now has an identity we can use induction to finish the proof as in Theorem 2 .

COROLlary [2, Corollary 7]. Let $R$ be a regular ring with identity and suppose that $R$ has bounded index. Let $\mathscr{L}$ be the set of lengths of primitive factor rings of $R$. Assume that if $m_{1} k_{1}+\cdots$ $+m_{t} k_{t}=k$ for some $k, k_{1}, \cdots, k_{t} \in \mathscr{L}$ and some positive integers $m_{1}, \cdots, m_{t}$ then $t=1$ and $k_{1}=k$. Then $R$ is a finite direct product of full matrix rings over strongly regular rings.

Proof. This now follows immediately from Theorem 3, Lemma 1 and [4, Theorem 4.2].

REMARKS. (1) Theorem 3 is false if $R$ does not have an iden- 
tity. To see this choose a field $F$ and let $R$ be the ring of all sequences in $M_{3}(F)$ which are eventually constant and of the form

$$
\left[\begin{array}{lll}
a & b & 0 \\
c & d & 0 \\
0 & 0 & 0
\end{array}\right] .
$$

(2) Theorem 3 is, however, still true if we assume that $R$, instead of being regular, is merely semisimple and $\pi$-regular. This may be seen by considering "pseudo-rank functions" on a $\pi$-regular ring (these functions should be defined as for regular rings [1, Chapter 16] except that the additional property " $N(x+y) \leqq N(x)+$ $N(y)$ for all $x, y$ " should be included). Suitable versions of Lemmas 2, 3 and 4 of [2] then follow after only minor modifications of their proofs. The details may safely be left to the reader.

Finally Theorem 2 can be improved even further if we already know that $R$ is biregular.

THEOREM 4. Let $R$ be a regular biregular ring of bounded index. Then $R$ is a direct product of homogeneous rings if and only if, for each $k$, no primitive factor ring of $J_{k+1} / J_{k}$ has length $m$ where $m \mid k$ and $m \neq k$.

Proof. Suppose no primitive factor ring of $J_{k+1} / J_{k}$ has length $m$ where $m \mid k$ and $m \neq k$. As $R$ is regular and biregular so is $J_{k+1} / J_{k}$. Using Lemma 1 and the remark following it, it is not hard to see that

$$
\cap\left\{P \in \operatorname{Spec}\left(J_{k+1} / J_{k}\right):\left(J_{k+1} / J_{k}\right) / P \text { has length } k\right\}=0 .
$$

By [2, Lemma 9] (if suitable care is taken, the proof in [2] is still valid even if $R$ does not have an identity), it follows that every primitive factor ring of $J_{k+1} / J_{k}$ has length dividing $k$. By our hypothesis $J_{k+1} / J_{k}$ must be homogeneous and so the result follows from Theorem 2.

COROLlaRy [2, Corollary 12]. Let $R$ be a regular biregular ring of bounded index and let $\mathscr{L}$ be the set of lengths of primitive factor rings of $R$. If $s \nmid t$ for all distinct $s, t \in \mathscr{L}$ then $R$ is a direct product of homogeneous rings.

Proof. This follows immediately from Theorem 4 and Lemma 1.

REMARK. In this instance there is nothing to be gained by replacing "regular" by " $\pi$-regular". Indeed by [4, Theorem 4.4] any 


\section{$\pi$-regular biregular ring of bounded index is regular anyway.}

\section{REFERENCES}

1. K. R. Goodearl, Von Neumann Regular Rings, London Pitman, 1979.

2. K. R. Goodearl and D. E. Handelman, Homogenization of regular rings of bounded index, Pacific J. Math., 84 (1979), 63-78.

3. N. Jacobson, Structure of Rings, Amer. Math. Soc. Colloq. Publ. No. 37, Providence, R. I. 1956.

4. I. Kaplansky, Topological representation of algebras II, Trans. Amer. Math. Soc., 68 (1950), 62-75.

Received May 27, 1980.

University of Melbourne

Parkville, Victoria 3052

Australia

Current address: University College

Belfield, Dublin 4, Ireland 


\section{PACIFIC JOURNAL OF MATHEMATICS}

\section{EDITORS}

DONALD BABBITT (Managing Editor)

University of Galifornia

Los Angeles, California 90024

Hugo RossI

University of Utah

Salt Lake City, UT 84112

C. C. MOORE AND ANDREW OGG

University of California

Berkeley, CA 94720

\section{J. DugundjI}

Department of Mathematics University of Southern California

Los Angeles, California 90007

R. Finn AND J. Milgram

Stanford University

Stanford, California 94305

\section{ASSOCIATE EDITORS}

R. ARENS

E. F. BECKENBACH

B. H. NeUmanN

F. WOLF

K. YosHIDA

\section{SUPPORTING INSTITUTIONS}

UNIVERSITY OF ARIZONA

UNIVERSITY OF BRITISH COLUMBIA

CALIFORNIA INSTITUTE OF TECHNOLOGY

UNIVERSITY OF CALIFORNIA

MONTANA STATE UNIVERSITY

UNIVERSITY OF NEVADA, RENO

NEW MEXICO STATE UNIVERSITY

OREGON STATE UNIVERSITY
UNIVERSITY OF OREGON

UNIVERSITY OF SOUTHERN CALIFONIA

STANFORD UNIVERSITY

UNIVERSITY OF HAWAII

UNIVERSITY OF TOKYO

UNIVERSITY OF UTAH

WASHINGTON STATE UNIVERSITY

UNIVERSITY OF WASHINGTON 


\section{Pacific Journal of Mathematics}

\section{Vol. 94, No. 1 \\ May, 1981}

Willy Brandal, Conditions for being an FGC domain $\ldots \ldots \ldots \ldots \ldots \ldots \ldots$

Allan Calder and Frank Williams, Incompressibility of maps and the

homotopy invariance of Čech cohomology $\ldots \ldots \ldots \ldots \ldots \ldots \ldots \ldots \ldots$

Jacques Chaumat, Quelques propriétés du couple d'espaces vectoriels

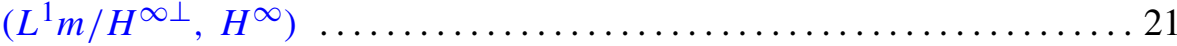

Manfred Droste and Rüdiger Göbel, Products of conjugate permutations . . 47

Jean Esterle, Rates of decrease of sequences of powers in commutative

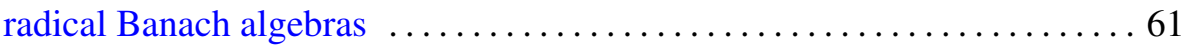

Allan Fryant, Ultraspherical expansions and pseudo analytic functions . . . 83

John Hannah, Homogenization of regular rings of bounded index. II . . . . . 107

Shigeru Haruki, On the theorem of S. Kakutani-M. Nagumo and J. L. Walsh

for the mean value property of harmonic and complex polynomials $\ldots .113$

Hugh M. Hilden, Representations of homology 3-spheres $\ldots \ldots \ldots \ldots \ldots 125$

Craig Huneke, A characterization of locally Macaulay completions . . . . . 131

Takesi Isiwata, Closed ultrafilters and realcompactness ................. 139

Joseph Weston Kitchen, Jr. and David A. Robbins, Tensor products of

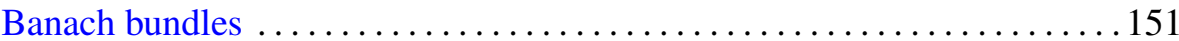

Allan J. Kroopnick, Note on bounded $L^{p}$-solutions of a generalized Liénard

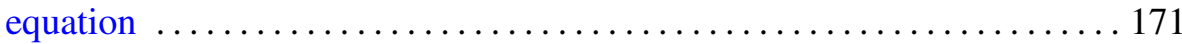

Ajay Kumar and Ajit Kaur Chilana, Spectral synthesis in products and quotients of hypergroups

Charles Livingston, Homology cobordisms of 3-manifolds, knot

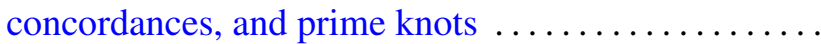

Hans Opolka, Projective representations of finite groups in cyclotomic fields

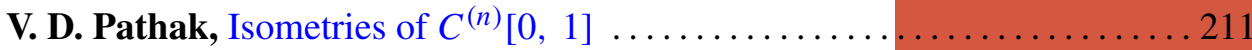

Mark Allan Pinsky, On the spectrum of Cartan-Hadamard manifolds . . . . . 2223

Judith Roitman, The number of automorphisms of an atomic Boolean

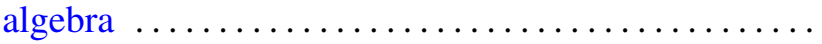

Kai Wang, Locally smooth torus group actions on integral cohomology complex projective spaces 